\title{
COVID-19 and myeloid cells: complex interplay correlates with lung severity
}

\author{
Franco R. D’Alessio' ${ }^{1}$ and Nicola M. Heller ${ }^{2}$ \\ 'Division of Pulmonary Critical Care Medicine, Johns Hopkins University School of Medicine, Baltimore, Maryland, USA. ${ }^{2}$ Department of Anesthesiology and Critical Care Medicine, Division of Allergy and \\ Clinical Immunology, Johns Hopkins University, Baltimore, Maryland, USA.
}

\begin{abstract}
COVID-19 spans a wide range of symptoms, sometimes with profound immune system involvement. How immune cell subsets change during the disease course and with disease severity needs further study. While myeloid cells have been shown to initiate and maintain responses to pneumonia and lung inflammation, often playing a role in resolution, their involvement with COVID-19 remains unknown. In this issue of the $J C I$, Sánchez-Cerrillo and Landete et al. investigated DCs and monocytes from blood and bronchial secretions of patients with varying COVID-19 severity and with healthy controls. The authors conclude that circulating monocytes and DCs migrate from the blood into the inflamed lungs. While sampling differences in sex, collection timing, bacteria/ fungal infection, and corticosteroid treatment limit interpretation, we believe that reprogramming monocyte or macrophages by targeting immunometabolism, epigenetics, or the cytokine milieu holds promise in resolving lung inflammation associated with COVID-19.
\end{abstract}

\section{Introduction}

COVID-19 disease morbidity and mortality have swept the globe since early 2020. The role of the immune response in SARS-CoV-2 pathogenesis and its determinants for the clinical course and outcomes remain elusive. An effective and coordinated immune response controls viral replication and limits immune pathology for a favorable clinical outcome (e.g., asymptomatic or a self-limiting respiratory viral infection). In contrast, an ineffective and dysregulated immune response could eventually control the virus, but sustained and excessive inflammation results in organ injury and an unfavorable outcome (e.g., respiratory failure, acute respiratory distress syndrome [ARDS], and multiorgan failure)

Approximately $14 \%$ of hospitalized patients with symptomatic COVID-19 infection develop severe disease with respiratory failure requiring intensive care unit support $(1,2)$. Clinical severity involves multiple systems, including respiratory, neurological, cardiovascular, hematological, renal, and even dermatological. Although other respiratory viruses, such as influenza, respiratory syncytial virus, other coronavirus, metapneumovirus, etc., can cause severe pneumonia and ARDS, SARS-CoV-2 differs in severity and duration, suggestive of hyperinflammation and/or impaired repair or resolution of inflammation.

As the scientific community elucidates the immunopathology of COVID-19, elevated proinflammatory cytokines and chemokines have led to the term cytokine storm. We and others feel this term is a misnomer, as other clinical syndromes, such as non-COVID ARDS, sepsis, and other cytokine release syndromes after CART-T therapy, are among clinical entities with

\section{Related Article: p. 6290}

Conflict of interest: The authors have declared that no conflict of interest exists.

similar cytokine profiles (3), yet these entities do not usually display a protracted ARDS course. Further, cytokine release syndrome secondary to CART-T therapy responds therapeutically to anti-IL-6. In addition to cytokine abnormalities and antibodies specific to COVID-19, dysregulated cellular immune responses include $\mathrm{CD}^{+} \mathrm{T}$ cell activation, hyperactivated or exhausted $\mathrm{CD}^{+} \mathrm{T}$ cells, decreased follicular helper cells, variable plasmablast responses (4), decreased regulatory $\mathrm{T}$ cells (5), decreased $\gamma \delta \mathrm{T}$ cells, increased NK cell activity, and increased neutrophil extracellular traps (6). Emerging literature also implies that myeloid cells play a role in COVID-19 severity (7). In this issue of the JCI, Sánchez-Cerrillo, Landete, and colleagues explore the role of myeloid cells in COVID-19 (8). The authors reveal the need for extensive profiling and evaluation of myeloid cell function and fate at different stages and severities of the disease.

\section{The role of myeloid cells in ARDS}

Myeloid cells including monocytes and macrophages initiate and maintain lung inflammatory responses to a variety of local or systemic insults (9); they also orchestrate resolution of lung inflammation (10). Research in mouse models of ARDS underscores the importance of monocytes that are recruited from blood. These bloodrecruited monocytes mature into monocyte-derived macrophages that are involved with lung repair and lung fibrosis. Similarly, resident lung macrophage populations also contribute at different phases of the repair/fibrosis processes. Notably, the literature regarding the role of monocytes and macrophages in human ARDS pathogenesis is mainly descriptive and speculative. Previous studies in ARDS patients show increases in total alveolar macrophages (AMs); specifically, surface and maturation markers implied that AMs are of monocytic origin and are proinflammatory (11, 


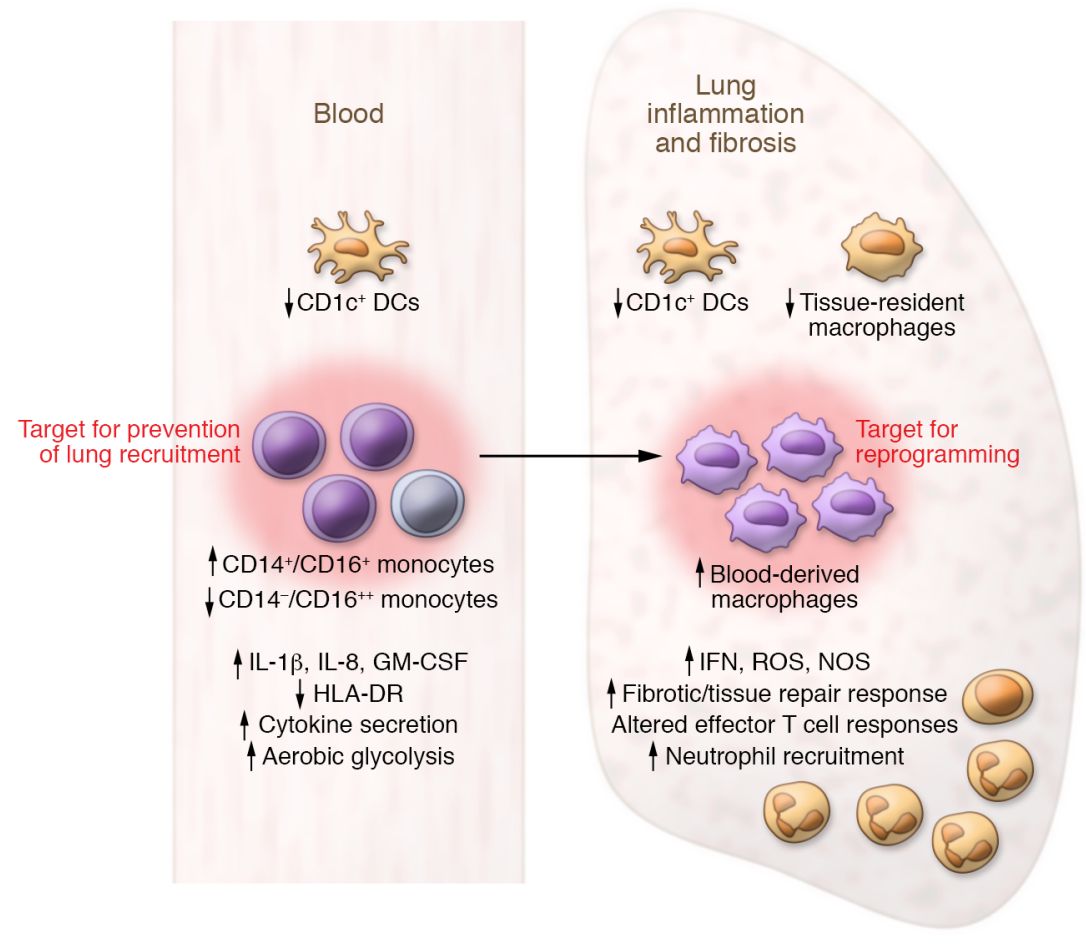

12). AMs from ARDS patients also secrete more IL-1 $\beta$ and IL-8, which correlate with increased mortality $(13,14)$. Transcriptionally, AMs and peripheral blood monocytes upregulate inflammatory genes, which correlates with worse outcomes (15). These data highlight the importance of immune cell tissue origin and suggest the need for comprehensive analysis of both blood and lung. One such mass cytometry analysis has revealed three unique AM populations in non-COVID ARDS that are defined by CD169 and PD-L1 expression. Notably, increased abundance of these three populations correlated with more ventilator-free days (16). Furthermore, a persistently activated AM transcriptional profile and lack of transition into a prorepair signature was associated with poor prognosis (15). However, clinical trials targeting macrophage function (GM-CSF [ref. 17] and IFN- $\beta$ [ref. 18]) in ARDS patients have failed. Blocking one target in an intricate milieu in ARDS is oversimplistic, and timing of intervention is pivotal. With the advent of high-throughput, high-dimensional flow cytometric, proteomic, and transcriptomic technologies, a better understanding of human monocyte/ macrophage subsets, origins, functions, and correlations with clinical outcomes in both COVID-19-induced and non-COVID19-induced ARDS is possible and critical.

\section{Monocytes and macrophages in COVID-19 disease}

Monocytes and lung macrophages are substantially altered, both in number and function, during COVID-19 disease. While disease severity affects the number and proportion of monocyte subsets in the blood and lung, some patterns emerge. Generally, in blood, the nonclassical monocyte subset $\left(\mathrm{CD} 14^{-} \mathrm{CD} 16^{++}\right)$is depleted, yet the intermediate subset $\left(\mathrm{CD} 14^{+} \mathrm{CD} 16^{+}\right)$ is expanded (19-22). The alveolar space is enriched for monocytes and monocyte-derived macrophages $(19,23)$, suggesting the lungs increase recruitment from the blood. Phenotypically, blood monocytes show diminished HLA-DR surface expression, possibly due to excess IL-6 exposure, suggesting reduced antigen-presenting capacity. Elevated complement receptor 3 (CD11b/CD18) expression (24) activates the cells to release cytokines. It is unclear whether blood monocytes and lung macrophages are directly infected by the virus or whether cellular changes are due to excessive inflammatory mediators. Functionally, monocytes from COVID-19 patients secrete the cytokine GM-CSF (25), although spontaneous IL-6 secretion varies by study $(25,26)$. The combination of these two cytokines switches monocyte differentiation from a DC fate
Figure 1. Model of changes in myeloid subsets during severe COVID-19. Sánchez-Cerrillo and Landete et al. (8) showed that in patients with severe COVID-19, circulating intermediate monocytes CD14+CD16+ increased and nonclassical CD14-CD16 ${ }^{++}$monocytes decreased. Corresponding surface markers CD11b, CD18, $\mathrm{CD}^{+} 0^{+}$(M1), CD206, CD69, and tissue factor CD142 all increased; the MHC class II cell surface receptor HLA-DR decreased. DCs decreased both in the blood and the lung. Cytokine secretion also increased, and phagocytosis was impaired. Within the lung, tissue-resident macrophages decreased, while mononuclear phagocytes derived from blood increased (8). Targeting circulating monocytes or lung-residing macrophages therapeutically may resolve inflammation, mitigate fibrosis, and repair lung damage.

to an inflammatory macrophage phenotype, consistent with the phenotyping of cells collected from the lower respiratory airway of COVID-19 patients $(8,23)$. Monocytes from COVID-19 patients also spontaneously secrete IL-1 $\beta$ and IL-8 (26). Excessive cytokine secretion is supported energetically by a switch to aerobic glycolysis (27). Transcriptional profiling has revealed a type I IFN gene signature, overexpression of genes that promote reactive oxygen and nitrogen species formation, inflammation-related chemokines, and a tissue-repair phenotype (28). This gene signature is consistent with a robust antiviral response and fibrotic response, which likely contribute to the collateral tissue damage and fibrosis seen in later stages of this disease. Although direct evidence that human monocyte-macrophages participate in COVID-19 pathogenesis is lacking, the findings described above and others (29) strongly implicate these cells as key players in the lung inflammation and damage seen in the disease.

\section{Pulmonary redistribution of myeloid subpopulations and COVID-19 severity}

Sánchez-Cerrillo and Landete et al. (8) profiled immune cells from the blood and respiratory secretions of a cohort of 
patients with critical disease and compared them with those of patients having either mild or severe disease and also with those of healthy controls. With results similar to those of previous studies, the authors noted that patients' blood was depleted of classical and intermediate monocyte subsets and DCs, and that inflammatory intermediate and nonclassical monocytes, granulocytes, and $\mathrm{CD} 1 \mathrm{c}^{+}$ cDCs appeared in their lungs. Diminished blood monocytes correlated with increased inflammatory markers, procalcitonin, and $\mathrm{C}$ reactive protein, levels, but not with IL-6 and type I IFN (Figure 1). Exhausted effector $C D 8^{+} T$ cells were enriched in the lungs of patients with COVID-19 compared with their blood and the blood of healthy controls. The dominance of inflammatory monocytes over $\mathrm{CD}_{1} \mathrm{c}^{+} \mathrm{DCs}$ may explain the abundance of exhausted lung $\mathrm{CD} 8^{+} \mathrm{T}$ cells. By surveying monocyte and DC populations in both the blood and the lungs, the authors conclude that the depletion of circulating monocytes and DCs is a result of migration to the inflamed lungs. Expression of myeloid CD40, an activation marker, was increased on classical and nonclassical blood monocytes in mild, but reduced in critical, COVID-19 patients, although expression was not different from that of healthy controls (8).

There are important limitations of Sánchez-Cerrillo and Landete et al. (8) that need considering: (a) the subjects in the control arm were predominantly females. Sex and sex hormones influence macrophage phenotype and function, as we and others have previously demonstrated (30). (b) The timing of sample collection, and consequently immune profiling, after infection was delayed in the critical group. Myeloid cell activation and plasticity dynamically change over the course of the infection, and consistent collection intervals would address variability. (c) The presence of bacterial or fungal superinfection modulates myeloid responses and likely modifies antiviral immune function in the lungs. A control group with active bacterial pneumonia without COVID-19 infection would add specificity. (d) A large proportion of critically ill patients received combinations of biologicals (anti-IL-6 blockade) and corticosteroids, which alter macrophage subpopulations and their activation. The use of immunomodulators and corticosteroids influences immune response and should be omitted if possible. (e) Superior flow cytometric phenotype and analysis would have allowed accurate quantification of lung myeloid subsets. (f) Comparison with non-COVID ARDS would determine specificity of their findings.

\section{Outstanding biology and future directions}

Immune profiling and sequencing of myeloid subpopulations in blood and alveolar compartments will uncover how these immune cells participate in response to SARS-CoV-2 infections and their interplay with other immune cells, such as $\mathrm{T}$ cells and neutrophils, and with nonimmune cells, such as endothelial cells and alveolar epithelial cells. Direct infection of SARSCoV-2 in myeloid cells or phagocytosis of infected epithelial cells will need to be elucidated. Understanding the signaling pathways in infected macrophages could lead to therapeutic targets, including JAK and inflammasome inhibitors, which are currently being tested in clinical trials. A full understanding of how the cytokine milieu diminishes effective monocyte-macrophage responses to the virus is needed. Moreover, determining how risk factors that associate with severe disease, such as age, sex, obesity, and diabetes, affect monocyte-macrophage dynamics in COVID-19 disease, as well as how therapeutic trials modulate myeloid responses longitudinally, will be informative.

It is imperative when considering therapies that target myeloid responses, to account for factors including timing and stage of the infection (early vs. late) and nature of the pathway to be modulated (single cytokine or receptor vs. upstream signaling pathway). For instance, abrogating myeloid proinflammatory responses to COVID-19 could limit disease and progression to organ injury, but could also lead to super infection with bacterial and fungal pathogens.

The potential of monocyte-macrophage reprogramming (immunometabolically, epigenetically, or through cytokine modulation) to resolve lung inflammation and repair the damaged lung is an unexplored cellular approach that could treat patients with COVID-19 while the world and the scientific community wait for a protective and safe vaccine (Figure 1).

\section{Acknowledgments}

This work was supported by grants from the Department of Defense (DoD): W81XWH-16-1-0510 (to FRD) and DoD W81XWH-16-1-0509 (to NMH).

Address correspondence to: Nicola M. Heller, 720 Rutland Avenue, Ross 367, Baltimore, Maryland 21205, USA. Phone: 410.955.1743; Email: nheller@jhmi.edu.

1. Wu C, et al. Risk factors associated with acute respiratory distress syndrome and death in patients with coronavirus disease 2019 pneumonia in Wuhan, China. JAMA Intern Med. 2020;180(7):934-943.

2. Richardson S, et al. Presenting characteristics, comorbidities, and outcomes among 5700 patients hospitalized with COVID-19 in the New York city area. JAMA. 2020;323(20):2052-2059.

3. Wilson JG, et al. Cytokine profile in plasma of severe COVID-19 does not differ from ARDS and sepsis. JCI Insight. 2020;5(17):140289.

4. Mathew D, et al. Deep immune profiling of COVID-19 patients reveals distinct immunotypes with therapeutic implications. Science. 2020;369(6508):eabc8511.

5. Chen G, et al. Clinical and immunological features of severe and moderate coronavirus disease 2019. J Clin Invest. 2020;130(5):2620-2629.

6. Middleton EA, et al. Neutrophil extracellular traps contribute to immunothrombosis in COVID-19 acute respiratory distress syndrome. Blood. 2020;136(10):1169-1179.

7. Merad M, Martin JC. Pathological inflammation in patients with COVID-19: a key role for monocytes and macrophages. Nat Rev Immunol. 2020;20(6):366-362.

8. Sanchez-Cerrillo I, et al. COVID-19 severity associates with pulmonary redistribution of $\mathrm{CD} 1 \mathrm{c}^{+} \mathrm{DCs}$ and inflammatory transitional and nonclassical monocytes. J Clin Invest. 2020;130(12):6290-6300.

9. Aggarwal NR, King LS, D’Alessio FR. Diverse macrophage populations mediate acute lung inflammation and resolution. Am J Physiol Lung Cell Mol Physiol. 2014;306(8):L709-L725.

10. Watanabe S, Alexander M, Misharin AV, Budinger GRS. The role of macrophages in the resolution of inflammation. JClin Invest. 2019;129(7):2619-2628.

11. Rosseau S, et al. Phenotypic characterization of alveolar monocyte recruitment in acute respiratory distress syndrome. Am J Physiol Lung Cell Mol Physiol. 2000;279(1):L25-L35.

12. Brittan M, et al. A novel subpopulation of monocyte-like cells in the human lung after lipopolysaccharide inhalation. Eur Respir J. 2012;40(1):206-214.

13. Jacobs RF, Tabor DR, Burks AW, Campbell GD. Elevated interleukin-1 release by human alveolar macrophages during the adult respi- 
ratory distress syndrome. Am Rev Respir Dis. 1989;140(6):1686-1692.

14. Donnelly SC, et al. Interleukin-8 and development of adult respiratory distress syndrome in at-risk patient groups. Lancet. 1993;341(8846):643-647.

15. Morrell ED, et al. Alveolar macrophage transcriptional programs are associated with outcomes in acute respiratory distress syndrome. Am J Respir Crit Care Med. 2019;200(6):732-741.

16. Morrell ED, et al. Cytometry TOF identifies alveolar macrophage subtypes in acute respiratory distress syndrome. JCI Insight. 2018;3(10):99281.

17. Paine $\mathrm{R}$, et al. A randomized trial of recombinant human granulocyte-macrophage colony stimulating factor for patients with acute lung injury. Crit Care Med. 2012;40(1):90-97.

18. Ranieri VM, et al. Effect of intravenous interferon $\beta$-1a on death and days free from mechanical ventilation among patients with moderate to severe acute respiratory distress syndrome: a randomized clinical trial. JAMA. 2020;323(8):725-733.

19. Silvin A, et al. Elevated calprotectin and abnormal myeloid cell subsets discriminate severe from mild COVID-19. Cell. 2020;182(6):1401-1418.e18.

20. Schulte-Schrepping J, et al. Severe COVID-19 is marked by a dysregulated myeloid cell compartment. Cell. 2020;182(6):1419-1440.e23.

21. Gatti A, Radrizzani D, Viganò P, Mazzone A, Brando B. Decrease of non-classical and intermediate monocyte subsets in severe acute SARS-CoV-2 infection. Cytometry $A$. 2020;97(9):887-890.

22. Kuri-Cervantes L, et al. Comprehensive mapping of immune perturbations associated with severe COVID-19. Sci Immunol. 2020;5(49):eabd7114.

23. Liao M, et al. Single-cell landscape of bronchoalveolar immune cells in patients with COVID-19. Nat Med. 2020;26(6):842-844.

24. Gupta R, Gant VA, Williams B, Enver T. Increased complement receptor-3 levels in monocytes and granulocytes distinguish COVID-19 patients with pneumonia from those with mild symptoms. Int JInfect Dis. 2020;99:381-385.

25. Zhou Y, et al. Pathogenic T cells and inflammatory monocytes incite inflammatory storm in severe COVID-19 patients. Natl Sci Rev. 2020;7(6):998-1002.
26. Kahn R, et al. Mismatch between circulating cytokines and spontaneous cytokine production by leukocytes in hyperinflammatory COVID19 [published online ahead of print August 13, 2020]. J Leukoc Biol. https://doi.org/10.1002/ JLB.5COVBCR0720-310RR.

27. Codo AC, et al. Elevated glucose levels favor SARS-CoV-2 infection and monocyte response through a HIF- $\alpha /$ glycolysis-dependent axis. Cell Metab. 2020;32(3):437-446.

28. Zhou Z, et al. Overly exuberant innate immune response to SARS-CoV-2 infection [preprint]. SSRN website. https://ssrn.com/ abstract=3551623 or http://dx.doi.org/10.2139/ ssrn.3551623. Posted March 24, 2020. Accessed October 8, 2020.

29. Grant RA, et al. Alveolitis in severe SARS$\mathrm{CoV}-2$ pneumonia is driven by self-sustaining circuits between infected alveolar macrophages and T cells. bioRxiv. https://doi. org/10.1101/2020.08.05.238188. Posted August 5, 2020. Accessed October 8, 2020.

30. Keselman A, Fang X, White PB, Heller NM. Estrogen signaling contributes to sex differences in macrophage polarization during asthma. JImmunol. 2017;199(5):1573-1583. 